1 Universidade Federal do Ceará (UFC), Programa de Pós-Graduação em Saúde da Família - Sobral (CE),

Brasil.

marcosaguiar61@hotmail.

com

2 Universidade Estadual Vale do Acaraú (UVA)

Centro de Ciências da Saúde - Sobral (CE), Brasil. izabellemontalverne@gmail. com

3 Universidade Estadual Vale do Acaraú (UVA), Centro de Ciências da Saúde - Sobral (CE), Brasil. jamylledz@hotmail.com

4 Universidade Estadual Vale do Acaraú (UVA), Centro de Ciências da Saúde - Sobral (CE), Brasil. karolineebezerra@gmail.com

5 Universidade Estadual Vale do Acaraú (UVA),

Centro de Ciências da Saúde - Sobral (CE), Brasil. ismael.brioso@hotmail.com

\section{Oficina Mapa Vivo na atenção básica: estratégia de planejamento local ao combate ao Aedes aegypti}

\author{
Workshop Live Map in primary care: local planning strategy to fight \\ Aedes aegypti
}

Marcos Aguiar Ribeiro', Izabelle Mont'Alverne Napoleão Albuquerque ${ }^{\mathbf{2}}$, Jamylle Lucas Diniz ${ }^{\mathbf{3}}$, Ana Karoline Barros Bezerra4 ${ }^{4}$ Ismael Brioso Bastos 5

RESUMO Este estudo objetivou relatar a realização de uma oficina do Mapa Vivo na atenção primária com enfoque no combate ao mosquito Aedes aegypti. Trata-se do relato da experiência de monitores e preceptores do Programa de Educação pelo Trabalho para a Saúde (PETSaúde/GraduaSUS). A realização da oficina constituiu-se em ferramenta de promoção de um espaço de compartilhamento e sistematização da territorialização realizada, colaborando para o conhecimento do território, desafios e possibilidades de intervenções.

PALAVRAS-CHAVE Atenção Primária à Saúde. Aedes. Planejamento participativo.

\begin{abstract}
This study aimed at describing a workshop Live Map in primary care with the focus on combating the mosquito Aedes aegypti. This is the experience report of monitors and preceptors of the Labor Education Program for Health (PET-Saúde/GraduaSUS). The workshop was constituted as a promotional tool of sharing space and territorial systematization, contributing to the knowledge of the territory, its challenges and intervention possibilities.
\end{abstract}

KEYWORDS Primary Health Care. Aedes. Participative planning. 


\section{Introdução}

Nos últimos anos, houve grande expansão da Estratégia Saúde da Família (ESF) em todo o território nacional, sendo considerada prioritária na estruturação da atenção básica e porta de entrada preferencial do Sistema Único de Saúde (SUS). A ESF tem papel fundamental no primeiro contato e na coordenação do cuidado, devendo atuar em articulação com as outras redes de atenção com o suporte dos serviços de apoio diagnóstico, assistência especializada e hospitalar (FAUSTO ET AL., 2014).

Todavia, apesar das melhorias sanitárias e da organização da rede de saúde, o combate às doenças transmitidas por vetores constitui-se um desafio para a ESF. Nessa perspectiva, ao se considerarem as doenças transmitidas pelo Aedes aegypti um problema de saúde pública desafiador, tem lugar a Reunião Internacional para Implementação de Novas Alternativas para o Controle de Aedes aegypti no Brasil, realizada em fevereiro de 2016 sob a coordenação do Programa Nacional de Controle da Dengue (PNCD) do Ministério da Saúde, onde foram avaliadas tecnologias com potencial de aplicação nos municípios afetados, considerando a estrutura e as questões operacionais do controle de vetores no Brasil. Nessa reunião, foram sistematizadas algumas abordagens recomendadas para controle. Dentre elas, destaca-se o mapeamento de risco, onde é importante identificar, no território, as áreas que acumulam casos de dengue de forma persistente, de forma a privilegiar as intervenções nessas áreas (BRASIL, 2016; VALLE, 2016).

Nesse contexto, o território deve ser entendido como um espaço vivo, geograficamente delimitado e ocupado por população específica, instituída por identidades comuns, sejam elas culturais, sociais ou outras. Um município pode ser dividido em diversos territórios para a implantação das áreas de abrangência das equipes de unidades básicas em saúde da família. Cada território possui peculiaridades em relação a seus usuários e equipes, à sua capacidade de estrutura física e recursos financeiros, organização social, conflitos e contradições inerentes ao local em que se encontra inserido. Assim, as estratégias para a melhor condução dos sistemas de saúde terão que se adequar, necessariamente, a essas diferenças regionais, pois não existe um padrão único e imutável de gestão (BRASIL, 2009B).

O processo de territorialização constitui-se um dos elementos essenciais para implantação da ESF e tem o objetivo de aproximar os profissionais e trabalhadores da saúde da realidade do território, colaborando para o desenvolvimento de ações coerentes às necessidades locais. Assim, a territorialização é o caminho para fazer o reconhecimento do território vivo com vistas à organização do processo de trabalho e das práticas de saúde, uma vez que as ações de saúde são implantadas sobre base territorial detentora de delimitação espacial previamente determinada (MONKEN; BARCELLOS, 2005).

Nessa perspectiva, o planejamento é influenciado por fatores que constroem a identidade do território, fazendo uso do pensamento crítico para tomada de decisão e sendo essencial reconhecer o momento de se adaptar a situações que requerem soluções criativas para a estratégia metodológica a ser utilizada para o problema encontrado (GRECO, 2004).

Logo, o mapeamento participativo é utilizado como mediador à elaboração de mapas para identificação das necessidades de saúde da comunidade segundo a determinação social da doença, a multidisciplinaridade, a percepção de coletivos e a dinamicidade do território para sua construção. Esse mapeamento consiste num processo reflexivo e crítico, que incorpora não só as dimensões sociais, afetivas, simbólicas e culturais como também as transformações territoriais e do modo de vida advindas com a reestruturação produtiva e a questão ambiental no território estudado (PESSOA ET AL., 2013).

Nesse ínterim, vale salientar a importância da integração ensino-serviço-comunidade 
para que a Universidade consiga trazer benefícios tanto para o serviço como para a população. Diante disso, nota-se a relevância da estratégia de diversificação dos cenários do ensino-aprendizagem como forma de favorecer a integração a realidade social, as políticas sociais e o Sistema Único de Saúde (SUS) para a contextualização da aprendizagem, a problematização, o desenvolvimento de habilidades de negociação para decisões coletivas e para a participação como base da cidadania (MOYSÉS ET AL., 2003).

Nesse sentindo, o Ministério da Saúde criou o Programa de Educação pelo Trabalho para a Saúde (PET-Saúde/ GraduaSUS), que contempla projetos de mudanças curriculares alinhadas às Diretrizes Curriculares Nacionais (DCNs) para cursos de graduação na área da saúde; de qualificação dos processos de integração ensino-serviço-comunidade de forma articulada entre o SUS e instituições de ensino; e de articulação com projetos do Ministério da Saúde e Ministério da Educação ou outros projetos de âmbito local ou regional relacionados à integração ensino-serviço-comunidade. No município de Sobral, tal projeto está sendo realizado de forma colaborativa entre a Secretaria Municipal de Saúde, Universidade Estadual Vale do Acaraú e Universidade Federal do Ceará Campus Sobral nos anos de 2016 e 2017.

Nesse sentido, este estudo tem o objetivo de relatar a realização da oficina Mapa Vivo no âmbito da atenção básica como estratégia de planejamento local com enfoque no combate ao mosquito Aedes Aegypti.

\section{Material e métodos}

Trata-se do relato da experiência de monitores, preceptores e tutores do PET- Saúde/ GraduaSUS acerca do processo de elaboração do desenvolvimento da oficina Mapa Vivo. Os relatos de experiência são metodologias de observação sistemática da realidade sem o objetivo de testar hipóteses, embora estabeleçam relações entre os achados dessa realidade e as bases teóricas pertinentes (DYNIEWICZ, 2009).

O cenário da experiência foi o município de Sobral, interior do estado do Ceará, mais especificamente no território de abrangência de um Centro de Saúde da Família (CSF). O município conta atualmente com 56 equipes de Estratégia Saúde da Família (ESF) e sete equipes do Núcleo de Apoio à Saúde da Família (Nasf) distribuídas em 31 CSFs localizadas em áreas definidas como territórios, com cobertura assistencial de $78 \%$ da população, equivalente a 214.206 pessoas acompanhadas.

O território do CSF na qual foi desenvolvida a experiência possui população de 12.900 habitantes e conta com quatro equipes de saúde da família, uma equipe do Nasf e residentes do Programa de Residência Multiprofissional em Saúde da Família (RMSF). Neste território, as equipes de RMSF são compostas pelas categorias Educação Física, Psicologia, Fonoaudiologia, Terapia Ocupacional, Serviço Social, Nutrição e Fisioterapia.

Vale salientar, ainda, que a experiência advém do grupo tutorial PET-Saúde/ GraduaSUS da Universidade Estadual Vale do Acaraú (UVA), que abrange projetos que se proponham a desenvolver mudanças curriculares alinhadas às Diretrizes Curriculares Nacionais para cursos de graduação na área da saúde a partir da integração ensino-serviço. No ano de 2016, propôs-se a realização de oficinas, integrando ensino-serviço, para discussão do contexto epidemiológico e priorização das necessidades locais, bem como ampliação de espaços que permitam ao estudante desenvolver práticas interdisciplinares e interprofissionais.

Esse grupo tutorial é composto por cinco tutores docentes, cinco preceptores, profissionais da saúde vinculados à Secretaria Municipal de Saúde, dois monitores bolsistas e quatro monitores voluntários, estudantes 
do curso de enfermagem. Os encontros acontecem semanalmente e discutem temáticas do cronograma proposto.

No que concerne à coleta de informações para a sistematização desta experiência, utilizaram-se relatórios e anotações pessoais com relatos das observações, sentimentos e vivências, realizada no período de abril a julho de 2016.

Inicialmente, realizou-se a identificação das necessidades locais do sistema de saúde do município de Sobral (CE). Em seguida, a escuta da coordenação da atenção básica e outros atores do Sistema Saúde Escola de Sobral - CE, a partir do que se priorizou a dengue. Diante disso, viu-se a necessidade de integrar as ações do PET-Saúde/GraduaSUS com módulos do curso de graduação em enfermagem da UVA. Dessa forma, optou-se por articular os módulos transversais de Atenção Básica à Saúde I ao VII (ABS) com enfoque no Módulo ABS III, que tem como tema estruturante o diagnóstico local em saúde. A partir disso, organizou-se o I Seminário PET/GraduaSUS: Situação Epidemiológica das Doenças Transmitidas pelo Aedes aegypti em Sobral, cujo objetivo foi o de aproximar o serviço e a gestão da universidade a partir da apresentação da situação epidemiológica das doenças transmitidas pelo Aedes aegypti no município.

Diante dos dados apresentados no seminário sobre o contexto epidemiológico das doenças transmitidas pelo mosquito Aedes aegypti em Sobral, foi possível promover a oficina do mapa vivo em colaboração com a equipe de saúde do CSF. Sua realização obedeceu aos seguintes momentos: i) apresentação da proposta do PET-Saúde/GraduaSUS; ii) Roda de Conversa acerca do território, com exposição fotos e vídeos; iii) elaboração de mapas representativos da dinâmica social, ambiental, cultural e do trabalho no território divididos em subgrupos por área de abrangência; iv) apresentação dos mapas, problematização e propostas de intervenção, em plenária; v) avaliação do momento.

Assim, a oficina Mapa Vivo aconteceu na roda da unidade de saúde da família. As rodas das unidades básicas de saúde de Sobral (CE) constituem-se como potentes espaços de cogestão de coletivos e possuem características administrativa, pedagógica, terapêutica e política. Dessa forma, justifica-se a escolha desse espaço para a realização da oficina Mapa Vivo, uma vez que suas características possibilitam a corresponsabilização e construção coletiva (CAMPOS, 2010).

Ressalta-se que a oficina Mapa Vivo foi elaborada a partir metodologias participativas, o que permitiu o compartilhamento de informações e conhecimentos acerca do território, bem como o desenvolvimento dos participantes e o surgimento de ideias inovadoras no grupo. Participaram da oficina os profissionais e trabalhadores da equipe de saúde da família, profissionais do Nasf e da RMSF, estudantes de enfermagem do módulo de Atenção Básica à Saúde III e monitores, preceptores e tutores do PET-Saúde/GraduaSUS, estes, facilitadores do momento.

A partir de então, dividiram-se os trabalhadores em dois grupos, cada um portando um mapa do território com os imóveis com foco positivo do mosquito Aedes aegypti. Dessa forma, os grupos foram instigados a dar 'vida' ao mapa, preenchendo-o com os equipamentos sociais existentes naquele bairro, como também identificando os possíveis motivos para aumento do número de focos do Aedes aegypti naquele local, de forma a gerar estratégias de combate. Assim, ao final da construção do mapa, os membros do PET-Saúde/GraduaSUS puderam elaborar, junto à equipe multidisciplinar presente, uma agenda estratégica de intervenções a serem realizadas nos locais apontados com maior quantitativo de focos.

\section{Resultados e discussões}

Como a finalidade de colaborar para o combate aos focos do mosquito Aedes aegypti, e consequentemente, para o controle 
das doenças transmitidas por esse vetor, objetivou-se elucidar o processo de territorialização a partir da construção de um mapa participativo, de forma a possibilitar subsídios para o conhecimento das áreas de aglomerados de imóveis com foco positivo do mosquito e proposição de intervenções coerentes com a realidade local.

No contexto da ESF, o mapa constitui-se como uma ferramenta utilizada para o diagnóstico e planejamento de atividade de campo, promovendo a reflexão frente às diversas situações de saúde e a elaboração de intervenções coerentes com a problemática do território, no intuito de melhorar a qualidade da atenção à saúde.

O mapeamento configura-se uma estratégia de apoio à qualificação e gestão da informação no âmbito da atenção básica, uma vez que possibilita, a partir de uma dimensão espacial, monitorar e avaliar indicadores e informações em saúde no âmbito do SUS (PINTO; ROCHA, 2016).

Nessa perspectiva, evidencia-se que o desafio representado pela implantação do SUS exige, cada vez mais, a utilização de ferramentas e de tecnologias que facilitem a identificação dos principais problemas de saúde que acometem a comunidade, bem como a definição de intervenções capazes de resolvê-los. Salienta-se a experiência exitosa de planejamento participativo na área de atuação das equipes da ESF como meio e processo de construção de intervenções coerentes e de maior efetividade (COSTA ET AL., 2014).

Dessa forma, os mapas contiveram a referência geográfica dos imóveis com foco positivo do mosquito Aedes aegypti. A partir de então, os participantes foram instigados a 'dar vida' ao mapa a partir da identificação dos equipamentos sociais do território e outros espaços de cuidados organizados com a participação de profissionais de saúde. Esse momento constitui-se uma importante etapa da oficina Mapa Vivo, uma vez que o objetivo é identificar possíveis espaços potentes para o desenvolvimento de ações ou intervenções comunitárias de combate ao mosquito Aedes aegypti a partir da reflexão da dinâmica social do território. Assim, o mapa passa a incorporar o cenário multicultural de significados e heterogeneidades do território.

Neste sentido, passou-se a reconhecer o território como base das iniciativas de articulação intersetorial para o combate ao mosquito Aedes aegypti. Com isso, faz-se necessário que as equipes da ESF atuem na identificação de situações de risco de infestação, como também potencializem a consolidação das redes locais de combate ao mosquito. Todavia, para que tal perspectiva seja efetiva, é preciso que se compreenda o território não só como um espaço geográfico, material e econômico como também social, cultural, com suas dimensões simbólica e imaginária (SÁ, 2009).

Posteriormente, os participantes refletiram sobre a distribuição espacial dos imóveis com foco positivo do mosquito Aedes aegypti. A referência geográfica foi realizada a partir dos ciclos de monitoramento realizados pelo agente de combate às endemias, que encaminham ao final de cada ciclo um consolidado dos imóveis positivos bem como os tipos de recipientes onde foram encontrados os focos. A partir dessa informação, os dados receberam referência geográfica para análise espacial.

Tal mapa foi analisado pelos participantes, que identificaram as ruas com aglomerados de imóveis positivos. Nesse sentido, foram destacadas três ruas com maiores aglomerados. Essas ruas tinham como característica comum ser uma área descoberta pelos Agentes Comunitários de Saúde (ACS), fato que impossibilitou um trabalho mais potente de educação em saúde por parte dos ACS em âmbito domiciliar.

Chiaravalloti-Neto et al. (2006) mostraram que as atividades de controle desenvolvidas pelos ACS com base na ESF são efetivas, uma vez que aproximam a comunidade no controle vetorial a partir de ações integradas de educação em saúde, comunicação e mobilização social. 
Além disso, vale salientar que, no território em questão, foram identificados, no período de janeiro a julho de 2016, 183 imóveis com foco positivo do mosquito Aedes aegypti. Destes, 179 (97,81\%) foram encontrados em âmbito residencial. No que concerne aos recipientes onde foram encontrados os focos, destacam-se os recipientes de armazenamento de água, tais como tambor, balde, pote e bacia (SOBRAL, 2016).

Tais informações foram disponibilizadas no momento da oficina Mapa Vivo, de forma a possibilitar a compreensão ampliada da situação de infestação do mosquito no território. Vale salientar, que ao se analisarem os recipientes onde foram encontrados os focos do mosquito contatou-se a atual situação de abastecimento de água no território. As limitações do sistema fazem com que a população tenha o hábito de armazenar água, a fim de garantir o uso em situações de falta de abastecimento. Essa situação também foi descrita pelos ACS, uma vez que trouxeram as singularidades de sua microárea de atuação.

Estudo realizado por Libanio, Favoreto e Pinheiro (2014) com um grupo de doze ACS identificou que as diferenças ambientais, sociais e econômicas das famílias influenciam tanto as ações de combate ao vetor Aedes aegypti como a relação da vigilância com a educação em saúde. Por exemplo, ocorreu na concentração das moradias e na quantidade de materiais inservíveis presentes no ambiente domiciliar e urbano como na forma como os moradores se relacionam entre si, com os ACS da área e com o território.

Em seguida, os participantes construíram coletivamente uma agenda estratégica de intervenções, tendo como referência as discussões e reflexões disparadas pelo Mapa Vivo. Cada proposta de intervenção foi avaliada pelo coletivo, que definiu as datas e responsáveis.

Dentre as intervenções pactuadas na oficina, destacam-se: roda de quarteirão com o objetivo de alertar os moradores da comunidade para os malefícios e doenças causadas pelo mosquito e a importância da participação da população no controle dos focos; mutirão e panfletagem com o intuito de retirar itens e recipientes que possam acumular água e servir de criadouros para o mosquito, estimulando a adesão da comunidade no controle dos focos; montagem de estandes com o propósito de mobilizar os quarteirões onde foram encontrados maiores quantidades de focos, alertando a população para as consequências do mosquito e da presença de focos.

Neste ínterim, verificou-se que as intervenções propostas tiveram o enfoque comunitário. Isso reforça o papel da ESF em seu esforço permanente de comunicação e troca de experiências e de conhecimentos entre os integrantes da equipe e da comunidade assistida (BRASIL, 2001). Portanto, as abordagens comunitárias a partir de estratégias tais como as rodas de quarteirão e mutirão são essenciais para o controle do vetor, uma vez que a participação da sociedade civil é de fundamental importância para o combate à proliferação do mosquito (ROCHA; CÂNDIDO; DANTAS, 2014).

Com isso, ao reconhecer a complexidade dos fatores que favorecem a proliferação do mosquito Aedes aegypti, ressalta-se a importância de intervenções que ultrapassem o setor saúde. As diretrizes nacionais para prevenção e controle de epidemias de dengue propostas pelo Ministério da Saúde priorizam a descentralização das ações de controle e combate ao vetor transmissor para os municípios, por meio de ações integradas de educação em saúde, com ênfase no saneamento ambiental e na sensibilização da população (BRASIL, 2009A).

Desse modo, o mapa participativo constituiu-se uma importante estratégia para o planejamento e desenvolvimento das intervenções da equipe de saúde da família, como também do PET-Saúde/GraduaSUS, visto que as atividades do grupo são 
fundamentadas e coerentes com as realidades do território e as necessidades do serviço, enquadrando a participação do curso de graduação em enfermagem nesse processo.

Assim, o planejamento constitui-se um instrumento de gestão, exercendo forte influência sobre o compromisso das pessoas com os objetivos institucionais, numa atitude permanente da organização (TANCREDI; BARRIOS; FERREIRA, 1998; BRASIL, 2015).

Além disso, a construção do mapa proporcionou a sistematização das vivências dos vários territórios, possibilitando o diagnóstico situacional do bairro. Também, a oficina permitiu uma experiência pedagógica para os monitores e preceptores do programa e para os estudantes da graduação, uma vez que os integrantes do grupo tutorial puderam participar do processo de planejamento, execução e avaliação da oficina.

Dessa forma, a oficina Mapa Vivo possibilitou a compreensão de que o mapa não se constitui como algo estático, mas 'vivo', ou seja, em constante renovação com as necessidades do território frente às doenças transmitidas pelo mosquito Aedes aegypti. Assim, por meio da constante avaliação desse mapa, a ESF torna-se qualificada para o desenvolvimento de intervenções coerentes com a realidade local.

\section{Conclusões}

A utilização da oficina Mapa Vivo possibilitou subsídios para avaliação, planejamento e definição de prioridades frente ao combate ao mosquito Aedes aegypti. Diante disso, a construção participativa do mapa propiciou, a partir da integração ensino-serviço-comunidade, o exercício do diagnóstico da comunidade e o desenvolvimento de intervenções estratégicas.

Portanto, o mapeamento participativo consiste em um instrumento de gestão do cuidado no âmbito da ESF, capaz de nortear a organização dos processos de trabalho na equipe de saúde, pois considera o ambiente e o perfil populacional, bem como as singularidades do território e das famílias frente às situação de infestação do mosquito Aedes aegypti.

Vale salientar, ainda, que o PET-Saúde/ GraduaSUS facilitou a integração ensino-serviço-comunidade durante o processo construção do Mapa Vivo junto a uma equipe multidisciplinar. Acredita-se que esta experiência constituiu-se um processo de intenso e significativo aprendizado para os monitores, preceptores e tutores do programa, visto que os espaços de aprendizagem foram ampliados e potencializados a partir do desenvolvimento de práticas colaborativas e interprofissionais. 


\section{Referências}

BRASIL. Conselho Nacional de Secretários de Saúde. A Gestão do SUS. Brasília, DF: CONASS, 2015.

Ministério da Saúde. Diretrizes nacionais para prevenção e controle de epidemias de dengue. Brasília, DF: Ministério da Saúde, 2009a.

Ministério da Saúde. Guia prático da saúde da família. Brasília, DF: Ministério da Saúde, 2001.

Ministério da Saúde. Relatório da Reunião internacional para implementação de alternativas para o controle do Aedes aegypti no Brasil. Brasília, DF: Ministério da Saúde, 2016.

Ministério da Saúde. O SUS de A a Z: garantindo saúde nos municípios. 3. ed. Brasília, DF: Ministério da Saúde, 2009b.

CAMPOS, S. W. G. Um método para análise e co-gestão de coletivos: a construção do sujeito, a produção de um valor de uso e a democracia em instituições: o método da roda. São Paulo: Hucitec, 2000.

CHIARAVALLOTI-NETO, F. et al. Controle do dengue em uma área urbana do Brasil: avaliação do impacto do Programa Saúde da Família com relação ao programa tradicional de controle. Cadernos de Saúde Pública, Rio de Janeiro, v. 22, n. 5, p. 987-997, 2006. Disponível em: <http://www.scielosp.org/pdf/csp/v22n5/11.pdf > . Acesso em: 3 abr. 2016

COSTA, F. M. et al. Planejamento participativo: processo de interação entre serviço e comunidade na Estratégia Saúde da Família. Revista Norte Mineira de Enfermagem, Minas Gerais, v. 3, n. 2, p. 170-183, 2014.

DYNIEWICZ, A. M. Metodologia da pesquisa em saúde para iniciantes. São Caetano do Sul: Difusão, 2009.

FAUSTO, M. C. R. et al. A posição da Estratégia Saúde da Família na rede de atenção à saúde na perspectiva das equipes e usuários participantes do PMAQ-AB. Saúde em Debate, Rio de Janeiro, v. 38, n. esp., p. 13-33, 2014. Disponível em: <http://www.scielo.br/pdf/sdeb/ v38nspe/0103-1104-sdeb-38-spe-0013.pdf>. Acesso em: 3 abr. 2016.

GRECO, R. M. Ensinando a administração em enfermagem através da educação em saúde. Revista Brasileira de Enfermagem, Brasília, DF, v. 57, n. 4, p. 504-507, 2004.

LIBANIO, K. R.; FAVORETO, C. A. O.; PINHEIRO, R. Análise da integração da Vigilância Ambiental no controle da dengue com a Estratégia Saúde da Família: impacto nos saberes e práticas dos agentes comunitários de saúde. Physis, Rio de Janeiro, v. 24, n. 1, p. 147-163, 2014. Disponível em: <http://www.scielo.br/ pdf/physis/v24nl/0103-7331-physis-24-01-00147.pdf>. Acesso em: 3 abr. 2016.

MOYSÉS, S. T. et al. Humanizando a educação em odontologia. Revista da ABENO, São Paulo, v. 3, n. 1, p. 58-64, 2003. Disponível em: <http://www.universidadesaudavel.com.br/wp-content/uploads/ARTIGOS/ artigo\%201\%20ABENO.pdf>. Acesso em: abr. 2016.

MONKEN, M.; BARCELLOS, C. Vigilância em saúde e território utilizado: possibilidades teóricas e metodológicas. Cadernos de Saúde Pública, Rio de Janeiro, v. 21, n. 3, p. 898-906, 2005. Disponível em: <http://www.scielo.br/scielo.php?pid=S0102$-311 X 2005000300024 \&$ script $=$ sci_abstract\&tlng=pt $>$. Acesso em: 3 abr. 2016.

PESSOA, V. M. et al. Sentidos e métodos de territorialização na atenção primária à saúde. Ciência \& Saúde Coletiva, Rio de Janeiro, v. 18, n. 8, p. 2253-2262, 2013.

PINTO, L. F.; ROCHA, C. M. F. Inovações na Atenção Primária em Saúde: o uso de ferramentas de tecnologia de comunicação e informação para apoio à gestão local. Ciência \&t Saúde Coletiva, Rio de Janeiro, v. 21, n. 5, p. 1433-1448, 2016. Disponível em: <http://www.scielo.br/ scielo.php?pid=S1413-81232013000800009\&script $=$ sci abstract\&tlng=pt>. Acesso em: 3 abr. 2016.

ROCHA, D. C.; CÂNDIDO, G. A.; DANTAS, R. T.

Políticas Públicas para a saúde e o papel da atenção 
básica de saúde no controle e prevenção da dengue no país. Revista Movimentos Sociais e Dinâmicas Espaciais, Recife, v. 3, n. 2, p. 247-261, 2014. Disponível em: $<$ http://www.revista.ufpe.br/revistamseu/index.php/ revista/article/view/90>. Acesso em: 3 abr. 2016.

SÁ, M. C. A fraternidade em questão: um olhar psicossociológico sobre o cuidado e a humanização das práticas de saúde. Interface - Comunicação, Saúde, Educação, São Paulo, n. 13, v. 1, p. 651-664, 2009. Disponível em: <http://www.scielo.br/scielo. php?pid=S1414-32832009000500016\&script=sci_ abstract\&tlng=pt>. Acesso em: 3 abr. 2016.

SOBRAL. Coordenação da Atenção Primária à Saúde. Imóveis com foco positivo do Aedes aegypti. Sobral: Secretaria de Saúde, 2016.

TANCREDI, F. B.; BARRIOS, S. R. L.; FERREIRA, J. H. G. Planejamento em Saúde: Saúde e Cidadania para gestores municipais de serviços de saúde. São Paulo: Fundação Petrópolis, 1998.
TEIXEIRA, C. F.; PAIM, J. S.; VILASBOAS, A. L. SUS, modelos assistenciais e vigilância da saúde. Informe Epidemiológico do SUS, Brasília, DF, v. 7, n. 2, p. 7-28, 1998. Disponível em: <http://scielo.iec.pa.gov.br/scielo. php?pid=S0104-16731998000200002\&script=sci_abstract>. Acesso em: 3 abr. 2016.

VALLE, D. Sem bala mágica: cidadania e participação social no controle de Aedes aegypti. Epidemiologia e Serviços de Saúde, Brasília, DF, v. 25, n. 3, p. 629-632, 2016. Disponível em: <http://scielo.iec.pa.gov.br/scielo.php?script=sci_arttext\&pid =S1679-49742016000300629>. Acesso em: 3 abr. 2016.

Recebido para publicação em agosto de 2016

Versão final em dezembro de 2016

Conflito de interesses: inexistente

Suporte financeiro: não houve 